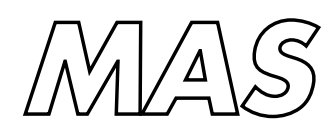

Modelling, Analysis and Simulation 
CWI is the National Research Institute for Mathematics and Computer Science. It is sponsored by the Netherlands Organization for Scientific Research (NWO).

CWI is a founding member of ERCIM, the European Research Consortium for Informatics and Mathematics.

CWI's research has a theme-oriented structure and is grouped into four clusters. Listed below are the names of the clusters and in parentheses their acronyms.

Probability, Networks and Algorithms (PNA)

Software Engineering (SEN)

Modelling, Analysis and Simulation (MAS)

Information Systems (INS)

Copyright (C) 2004, Stichting Centrum voor Wiskunde en Informatica

P.O. Box 94079, 1090 GB Amsterdam (NL)

Kruislaan 413, 1098 SJ Amsterdam (NL)

Telephone +31205929333

Telefax +31205924199

ISSN 1386-3703 


\title{
Branching of positive discharge streamers in air at varying pressures
}

\author{
ABSTRACT \\ The formation of positive streamers in a $17 \mathrm{~mm}$ gap in air is studied at pressures varying in the \\ range from 1010 mbar to 100 mbar. An intensified charge coupled device camera is used to \\ image the discharge. At high pressures, the discharge shows many branches, while at low \\ pressure fewer branches arise. The structure is not simply determined by the ratio of voltage \\ over pressure.
}

2000 Mathematics Subject Classification: 70-05

Keywords and Phrases: Corona ; gas discharges ; optical imaging ; sparkgap 


\title{
Branching of positive discharge streamers in air at varying pressures
}

\author{
Tanja M.P. Briels, Eddie M. van Veldhuizen, and Ute Ebert
}

\begin{abstract}
The formation of positive streamers in a $17 \mathrm{~mm}$ gap in air is studied at pressures varying in the range from 1010 mbar to 100 mbar. An intensified charge coupled device camera is used to image the discharge. At high pressures, the discharge shows many branches, while at low pressure fewer branches arise. The structure is not simply determined by the ratio of voltage over pressure.
\end{abstract}

Index terms - Corona, gas discharges, optical imaging, sparkgap.

Streamers are narrow rapidly growing channels, which arise when a high voltage is applied to a nonconducting medium like air. They form the early stages of sparks of all sizes. Recently they are observed at low-pressure in the atmosphere at $\sim 40$ to $90 \mathrm{~km}$ altitude, here they are called sprite discharges [1]. Since streamers are also used in industry, e.g. in water cleaning or photocopiers, a better understanding of the phenomena is desirable. Therefore, at TU/e experiments are performed on streamers [2, 3], while at CWI analytical research [4] and simulations [5] are done. We aim at a quantitative comparison between theory and experiment of different streamer parameters.

The measurements are performed in a $17 \mathrm{~mm}$ pointplane gap in air. A high voltage power supply charges a 250 $\mathrm{pF}$ capacitor via a $5 \mathrm{M} \Omega$ resistor. This capacitor discharges over the point-plane gap when the electric circuit is closed via a switch. There are two types of switches that can be used, a semiconductor switch and a sparkgap [2]. In the present measurements a sparkgap is used, which leads to a voltage pulse at the anode with a rise time of $10 \mathrm{~ns}$.

The pictures are made with a fast ICCD-camera (Andor DH534-18), which has a minimum optical gate of 0.8 ns and a spatial resolution of $21 \mu \mathrm{m}$. Here a long illumination time of $10 \mu_{\mathrm{s}}$ is used. For comparison, typical streamer propagation times at 1 bar are $\sim 100$ ns. Furthermore, the images are made with different gains to obtain the clearest patterns and not to overexpose the camera. Absolute intensities in pictures with different gains are difficult to compare.

Figure 1 shows pictures of positive discharges at a pressure of 1010 mbar (a), 400 mbar (b), 200 mbar (c) and
100 mbar (d) at different voltages. The leftmost column shows the lowest voltage at which discharges occur at the respective pressure.

When comparing the pictures, it is observed that at high pressure the discharge forms more and thinner streamers than at low pressure. At 1010 mbar, their number also increases with voltage while at lower pressures their number remains constant, i.e. four streamers at 400 mbar, two at 200 mbar and one at 100 mbar. Furthermore, at 1010 mbar, streamers at low voltages do not bridge the gap, while at lower pressures they always do.

The pictures also show that the structures are not simply determined by $U / p$. If this would be the case, the picture at $20 \mathrm{kV}$ and 1010 mbar should be comparable to the one at $8 \mathrm{kV}$ and $400 \mathrm{mbar}$, which is not the case, or to a discharge at e.g. $4 \mathrm{kV}$ and 200 mbar, which does not exist. This non-linearity is also found when comparing [3] and [6]. In [3] a discharge is found in argon at 1000 mbar and a lowest voltage of $3.5 \mathrm{kV}$. In [6] measurements are done on argon at $4.65 \mathrm{mbar}$ and $410 \mathrm{~V}$. Hence the pressures differ a factor of $\sim 200$ and the voltage only a factor of $\sim 10$.

\section{REFERENCES}

[1] N. Liu, V. P. Pasko, "Effects of photoionization on propagation and branching of positive and negative streamers in sprites", J. Geophys. Res. 109, A04301 (2004).

[2] E.M. van Veldhuizen, P.C.M. Kemps, W.R. Rutgers, "Streamer branching in a short gap: The influence of the power supply", IEEE Trans. Pl. Sc. 30, 162-163 (2002).

[3] E.M. van Veldhuizen, W.R. Rutgers, "Pulsed positive corona streamer propagation and branching", J. Phys. D.: Appl. Phys. 35, 2823-2829 (2002).

[4] B. Meulenbroek, A. Rocco, U. Ebert, "Streamer branching rationalized by conformal mapping techniques", Phys. Rev. E 69, 067402 (2004).

[5] C. Montijn, B. Meulenbroek, U. Ebert, W. Hundsdorfer, "Numerical simulations and conformal analysis of growing and branching negative discharge streamers", IEEE Trans. Pl. Sc., submitted (2005).

[6] E. Wagenaars, M.D. Bowden, G.M.W. Kroesen, "Plasma emission imaging of electrical breakdown in low-pressure argon”, IEEE Trans. Pl. Sc., submitted (2005).

Manuscript received July 1, 2004.

The authors are with the Department of Applied Physics, Eindhoven University of Technology (TU/e), P.O. Box 513, 5600 MB Eindhoven, The Netherlands (email: T.M.P.Briels@tue.nl).

U. Ebert is also with the Center for Mathematics and Computer Science (CWI), P.O. Box 94079, 1090 GB Amsterdam, The Netherlands.

Publisher Identifier S XXXX-XXXXXXX-X 

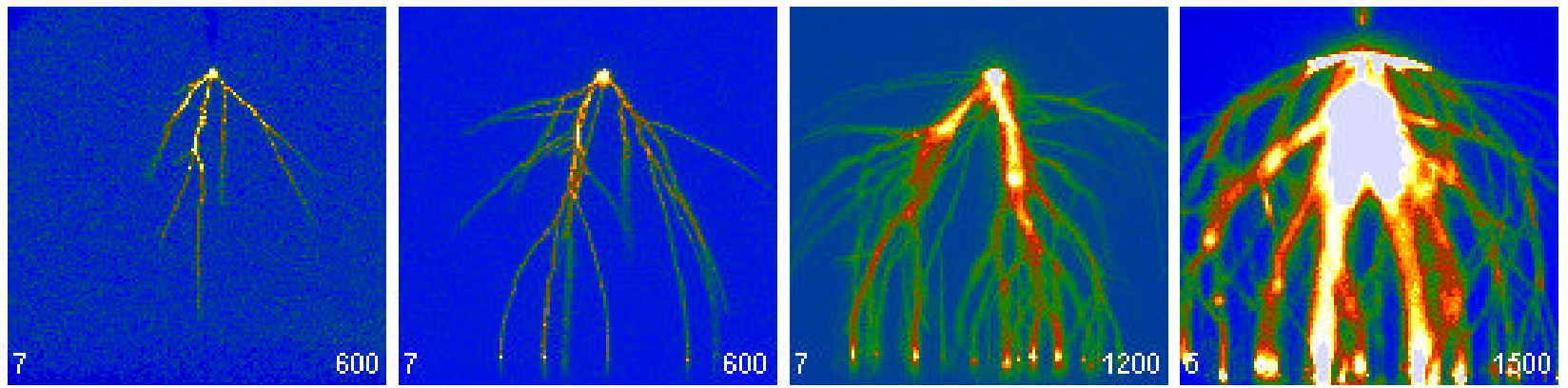

[a]
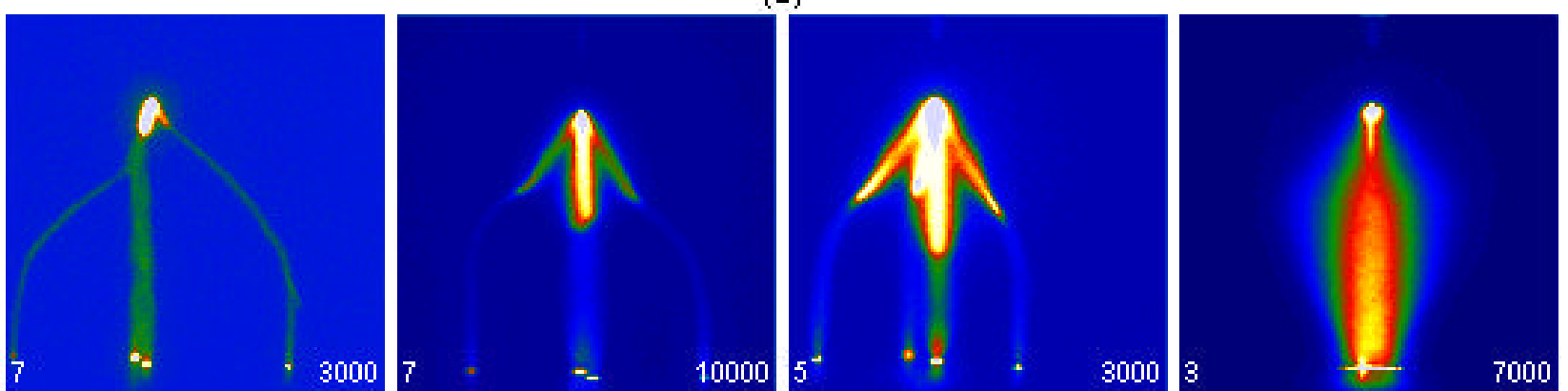

(b)
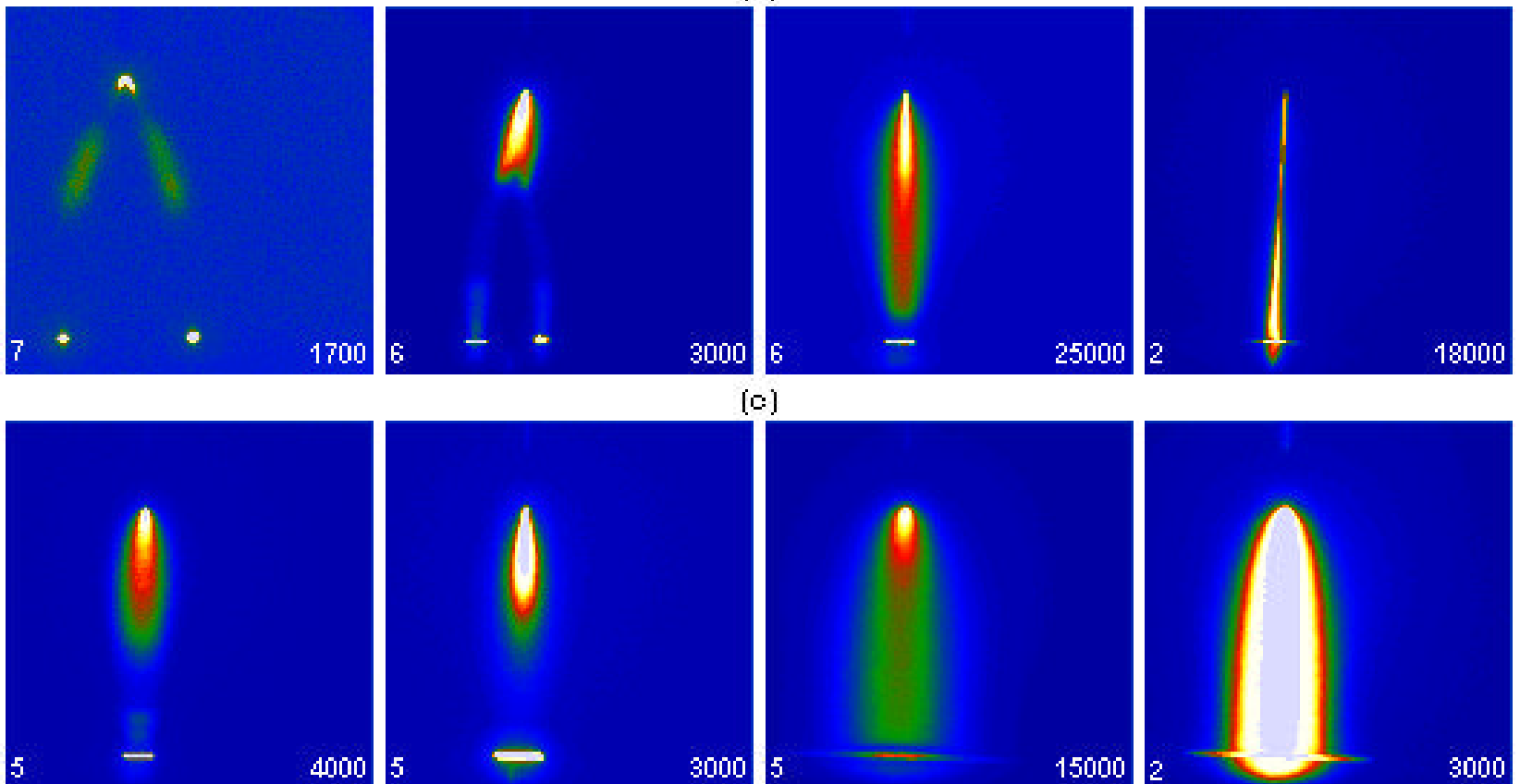

(c)
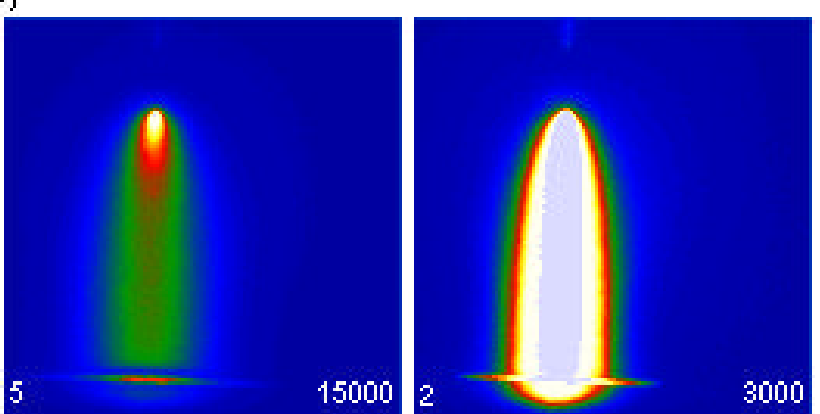

(d)

Lowest intensity $(\sim 0)$

Highest intensity

Figure 1: The ICCD images are time integrated pictures $(10 \mu \mathrm{s})$ of the gas discharge at different pressures and voltages. The discharge starts at the positive anode tip and ends at the negative cathode plate, $17 \mathrm{~mm}$ below the tip. From left to right (a) $8 \mathrm{kV}, 10 \mathrm{kV}, 15 \mathrm{kV}, 21 \mathrm{kV}$ at a pressure of 1010 mbar, (b) $6 \mathrm{kV}, 8 \mathrm{kV}, 10 \mathrm{kV}, 15 \mathrm{kV}$ at 400 mbar, (c) $5 \mathrm{kV}, 5 \mathrm{kV}, 7 \mathrm{kV}, 7 \mathrm{kV}$ at $200 \mathrm{mbar}$ and (d) $3 \mathrm{kV}, 4 \mathrm{kV}, 7 \mathrm{kV}, 7 \mathrm{kV}$ at 100 mbar. The gain of the ICCD-camera is given on the left hand side of the pictures, the maximum intensity of the pictures at this gain is given on the right hand side. The relative intensities are indicated by the colour bar, except in the first three pictures of (a) where blue indicates an intensity of $\sim 300$ (instead of 0 ) and in the first picture of (c) where blue denotes $\sim 800$. The last picture of (b) and the last two pictures of (c) and (d) are sparks and are visible by naked eye. The other images are streamers. 